\title{
Triptolide inhibits the progression of atherosclerosis in apolipoprotein $\mathrm{E}^{-/-}$mice
}

\author{
LONGFENG LUO and TIANLUN YANG
}

Department of Cardiovascular Medicine, Xiangya Hospital of Central South University, Changsha, Hunan 410008, P.R. China

Received June 11, 2015; Accepted June 7, 2016

DOI: $10.3892 /$ etm.2016.3619

\begin{abstract}
Atherosclerosis, the major cause of cardiovascular disease, is accompanied by a chronic inflammatory response during the disease. Triptolide (TPL) is an active natural compound that has been demonstrated to possess anti-inflammatory activities in various cell types. However, the effects of TPL on atherosclerosis have not yet been studied. The goal of the present study was to determine the effects of TPL on apolipoprotein E knock-out (ApoE $\%$ ) mice fed with a high-fat diet and to analyze the changes in lipid metabolism and inflammatory cytokines to clarify the underlying molecular mechanisms. Firstly, the genotypes of ApoE $/$ mice and corresponding wild-type mice were identified using polymerase chain reaction. The ApoE $\%$ mice were randomly divided into four groups: ApoE ${ }^{-/}$model mice, and $\mathrm{ApoE}^{-/}$mice treated with 25,50 or $100 \mu \mathrm{g} / \mathrm{kg}$ TPL every twice day. Wild-type mice with the same genetic background constituted the fifth group. The mice in each group were given a high-fat diet from week 8 after birth until week 20. Total cholesterol and total triglyceride levels were determined at 16 and 20 weeks. The results demonstrated that the levels of total cholesterol and total triglyceride in the plasma were highly increased in ApoE $\%$ mice models, compared with those of wild-type mice, and the ApoE $\%$ mice treated with TPL had decreased levels of total cholesterol and total triglyceride in plasma, which exhibited a dose-dependent reduction as the dose of TPL increased. Moreover, the effects of TPL on the production of inflammatory cytokines in macrophages were determined by ELISA. The results demonstrated that the macrophages from $\mathrm{ApoE}^{-}$ mice produced high levels of the inflammatory cytokines tumor necrosis factor- $\alpha$, interleukin (IL)-1 $\beta$, IL- 6 and IL-8. However, following treatment with TPL doses of 25, 50 and $100 \mu \mathrm{g} / \mathrm{kg}$, the cytokine levels were significantly decreased in a dose-dependent manner. Additionally, proteins associated with
\end{abstract}

Correspondence to: Dr Longfeng Luo, Department of Cardiovascular Medicine, Xiangya Hospital of Central South University, 87 Xiangya Road, Changsha, Hunan 410008, P.R. China E-mail: luoalongfeng@163.com

Key words: atherosclerosis, triptolide, inflammatory cytokines, anti-ATP-binding cassette transporter A1, liver $\mathrm{X}$ receptor lipid metabolism were tested by western blotting. The results showed that the expression of anti-ATP-binding cassette transporter A1 in the macrophages of $\mathrm{ApoE}^{-/}$mice was increased following treatment with TPL. However, the expression levels of LXR $\alpha$ were not markedly changed following treatment of the mice with different doses of TPL. These results suggest that TPL inhibited the progression of atherosclerosis not only by inhibiting the chronic inflammatory response, but also by regulating lipid metabolism, which may provide new insights useful in the clinical therapy of atherosclerosis.

\section{Introduction}

Atherosclerosis is the main underlying cause of many cardiovascular diseases, and has seriously harmful effects on human health $(1,2)$. As living standards improve, the incidence of cardiovascular and cerebrovascular disease is increasing. Atherosclerosis is a most important and commonly observed vascular disease, with the characteristic of initiation from the intima in the affected artery (3). Lesion development usually involves the initial accumulation of lipids and complex carbohydrates, followed by bleeding, thrombus formation, fibrosis and calcinosis (4). Gradually, the artery becomes calcified and the elasticity of medium and large arteries is lost $(5,6)$. Once the lesions have developed to a size large enough to block the arterial lumen, the tissues or organs that are supplied by the arteries become ischemic or necrotic $(7,8)$. There are several hypotheses for the cause of atherosclerosis, including lipid infiltration, smooth muscle cell (SMC) proliferation, and the thrombosis source theory (9-11). However, to date, the pathogenesis of atherosclerosis has not been clearly explained and clarified. However, investigation of the mechanism has revealed that atherosclerosis is always accompanied by an inflammatory response during the progression of the disease. Thus, Ross proposed that atherosclerosis is an inflammatory disease $(12,13)$.

Apolipoprotein E (ApoE) is a glycoprotein that was first identified in 1973 as a constituent of very-low-density lipoprotein (VLDL) in normal humans. It is a single peptide of 299 amino acids with a molecular weight of $34 \mathrm{kDa}$, and has subsequently been found in all lipoprotein classes (14-16). ApoE is an important component of plasma apolipoprotein. In humans, ApoE is mainly present in VLDL, low-density lipoprotein (LDL), chylomicrons (CMs) and CM remnants. It is present in the subclasses of $\beta$-VLDL and high-density lipoprotein. 
In 1992, Shimano et al first established a transgenic mouse expressing rat ApoE, in which a $>4$-fold higher level of plasma ApoE, and significantly lower concentrations of VLDL and LDL cholesterol were observed, when compared with normal mice $(17,18)$. Moreover, the animals with a high concentration of ApoE exhibited an alleviation of high-fat diet-induced hypercholesterolemia. Conversely, the ApoE knock-out mouse model was also established in 1992 (19,20). Mice homozygous for the ApoE mutation can normally survive and have reproductive ability. If ApoE-deficient mice are fed with a high-fat diet, the level of cholesterol can become elevated to as high as $46.5 \mathrm{mmol} / 1$ in the plasma, mainly distributed in VLDL and intermediate-density lipoproteins, suggesting that the mice seriously lack the cholesterol-removing ability of ApoE lipoprotein and the ApoE receptor-binding function $(21,22)$. Atherosclerotic plaques undergo a $>3$-fold increase in size when a high-fat diet is administered for $>1$ month (22). The pathological features and histological distributions in these model mice are very similar to those in human atherosclerotic disease, which suggests that ApoE plays an important role in the pathogenesis of coronary heart disease in humans $(23,24)$. Thus, in the present study, ApoE knock-out mice were used as a model of atherosclerosis in which to investigate the effects of triptolide (TPL) on the pathogenesis of this condition.

TPL is an active compound extracted from the Chinese medicinal herb Tripterygium wilfordii Hook.f., which has been demonstrated to possess anti-inflammatory activities in various cells $(25,26)$. However, the effects of TPL on atherosclerosis have not yet been investigated. Thus, the goal of the present study was to determine the effects of TPL on ApoE $\%$ mice with a high-fat diet and to analyze the changes of proteins associated with lipid metabolism and inflammatory cytokines to clarify the underlying molecular mechanisms.

\section{Materials and methods}

Antibodies. Anti-ATP-binding cassette transporter A1 (ABCA1) antibody (cat. no. NB400-105) was obtained from Novus Biologicals, LLC (Littleton, CO, USA). Primary rabbit polyclonal LXR $\alpha$ (cat. no. ab3585) was purchased from Abcam (Cambridge, UK). Primary mouse monoclonal $\beta$-actin antibody (cat. no. sc-47778) and secondary antibodies, including horseradish peroxidase (HRP)-conjugated goat anti-rabbit IgG (cat. no. sc-2004) and goat anti-mouse IgG-HRP (cat. no. sc-2005) were purchased from Santa Cruz Biotechnology, Inc. (Dallas, TX, USA).

Animals. A total of 12 eight-week-old ApoE knock-out $\left(\mathrm{ApoE}^{-/}\right)$mice (male, 5; female, 7; weight, 18-20 g) were obtained from the Laboratory Animal Center of Peking University Health Science Center (Beijing, China) and housed in specific pathogen-free conditions. The $\mathrm{ApoE}^{-/}$mice were given a high-fat diet from weeks 8-20, and were used as atherosclerosis models. In addition, 30 8-week-old male wild-type C57BL/6 mice, weighing 18-20 g, with the same genetic background were used as blank controls. The feed was constructed with $21 \%$ fat, $0.15 \%$ cholesterol and $19.5 \%$ casein. All mice were free to eat and drink and the ambient temperature was kept at $22 \pm 2^{\circ} \mathrm{C}$ with a humidity of $50-60 \%$. The lighting was artificially controlled in the room and alternating cycles of
12 h-light (8:00-20:00) and 12 h-darkness (20:00-8:00 the next day) were used.

Genotype identification of the ApoE ${ }^{-/}$mice. The tail tips of the mice were cut and DNA samples were prepared using a DNA extraction kit (Bioteke Corporation, Beijing, China). The genotype of ApoE was detected by polymerase chain reaction (PCR) assay using an 2X EasyTaq PCR SuperMix (Quanshijin Corp., Beijing, China). Three PCR primers were used as follows: Primer 1: 5'-GCCTAGCCGAGGGAGAGC CG-3'; primer 2: 5'-TGTGACTTGGGAGCTCTGCAGC-3'; and primer 3: 5'-GCCGCCCCGACTGCATCT-3'.

Thermal cycling conditions were $95^{\circ} \mathrm{C}$ for $10 \mathrm{~min}$, followed by 40 cycles of $30 \mathrm{sec}$ at $95^{\circ} \mathrm{C}, 30 \mathrm{sec}$ at $67^{\circ} \mathrm{C}$ and $30 \mathrm{sec}$ at $72^{\circ} \mathrm{C}$, and then $10 \mathrm{~min}$ at $72^{\circ} \mathrm{C}$ for final elongation. The length of the DNA fragments of wild-type, ApoE $/$ type and the heterozygous type mice are expected to be $155 \mathrm{bp} / 155 \mathrm{bp}$, $245 \mathrm{bp} / 155 \mathrm{bp}$ and $155 \mathrm{bp} / 155 \mathrm{bp}$, respectively.

Western blot analysis. Cells were collected and lyzed using RIPA buffer. Total protein ( $30 \mu \mathrm{g} /$ well) was separated by SDS-PAGE and transferred to nitrocellulose membrane at $400 \mathrm{~mA}$ for $2 \mathrm{~h}$. The membrane was blocked with 5\% skim milk at room temperature prior to incubation with the primary antibodies (all 1:1,000) overnight at $4^{\circ} \mathrm{C}$. Subsequently, the membrane was washed three times with $1 \mathrm{X}$ Tris-buffered saline with Tween 40 (0.1\%; TBST) for $5 \mathrm{~min}$ and incubated with the secondary antibodies for $30 \mathrm{~min}$ at room temperature. The membrane was washed three times with TBST and the bands were developed using an enhanced chemiluminescence kit. BioRad GelDoc XR and Quantity One 1-D analysis software (Bio-Rad Laboratories, Inc., Hercules, CA, USA) were used for band detection and visualization, respectively.

Animal experiment. The 8-week-old male ApoE $\%$ mice and C57BL/6 mice (control group) were housed in specific pathogen-free conditions. The $\mathrm{ApoE}^{-/-}$mice were randomly divided into four groups: ApoE ${ }^{-/-}$model (untreated) and three TPL treatment groups. The mice in the TPL groups were intraperitoneally injected with 25,50 or $100 \mu \mathrm{g} / \mathrm{kg}$ TPL every 3 days, from week 12 to week 20 after birth. Each group contained 6 mice.

Measurement of total cholesterol and total triglyceride levels in plasma. The mice were divided into five groups and were treated as described above. At weeks 16 and 20, blood samples were collected from the tails of the mice. Total cholesterol and total triglyceride levels in plasma were determined using kits from Mingfeng Biotechnology Co., Ltd. (Shanghai, China) according to the protocols provided with the kits.

Cytokine production by macrophages. Mice were sacrificed via cervical dislocation and $5 \mathrm{ml}$ of 1640 medium was subsequently injected into the abdominal cavity with a syringe to harvest peritoneal fluid. Following centrifugation at $200 \mathrm{rpm}$ for $10 \mathrm{~min}$, the macrophages were isolated. Peritoneal macrophages from the five groups were prepared and adjusted to $3 \times 10^{5}$ cells $/ \mathrm{ml}$ with 1640 medium for stimulation by TPL. After adherence, the cells were treated with $25 \mu \mathrm{g} / \mathrm{ml}$ oxidized LDL (oxLDL; Luwen Biocorporation, Shanghai, China) for 
$48 \mathrm{~h}$. The supernatants were harvested and the concentration of inflammatory cytokines, namely tumor necrosis factor (TNF)- $\alpha$, interleukin (IL)-1 $\beta$, IL-6 and IL-8, were determined with enzyme-linked immunosorbent assay (ELISA) kits (Neobioscience, Beijing, China) according to the manufacturer's protocol and a Benchmark Microplate Reader (Bio-Rad Laboratories, Inc.). In the present experiment, all samples were analyzed in duplicate for determination of the cytokine levels.

Statistical analysis. The data were analyzed using SPSS 20.0 software (IBM SPSS, Armonk, NJ, USA). Cytokine production data were compared using Student's t-tests. Data are expressed as means \pm standard errors of the means. $\mathrm{P}<0.05$ was considered to indicate a statistically significant difference.

\section{Results}

ApoE $\%$ mice are identified and selected by PCR. Mouse ApoE primers were used to identify the wild-type allele (155 bp) and ApoE null allele (245 bp). The heterozygote with wild-type and ApoE null alleles had a mixture of 155-bp and 245-bp fragments. Homozygotes of wild-type mice only had the wild-type allele of $155 \mathrm{bp}$ and homozygotes of knock-out mice only had the ApoE null allele of 245 bp. Two male ApoE $\%$ mice (lanes 1 and 2) and four female ApoE\% mice (lanes 2, 3, 5 and 7) were screened. Additionally, the corresponding wild-type mice were also identified with same genetic background to ApoE $\%$ mice. PCR results are shown in Fig. 1.

Body weights of ApoE- knock-out mice are lower than those of mice in the other groups. Body weight is a key indicator of toxicity for drug-treated mice. Mice in the different groups were given a high-fat diet from week 8 after birth until week 20, and were allowed to eat and drink freely. The body weights of the mice were determined once every week during the high-fat diet. As shown in Fig. 2, the body weights of the mice in the ApoE ${ }^{-/}$knock-out group were gradually and significantly decreased compared with those in the wild-type control group $(\mathrm{P}<0.01)$. However, ApoE $/-$ mice treated with 25 or $50 \mu \mathrm{g} / \mathrm{kg}$ TPL exhibited no marked variation in body weight compared with the wild-type control group. Notably, the body weights of the ApoE ${ }^{-/}$mice treated with $100 \mu \mathrm{g} / \mathrm{kg}$ TPL were less than those of the wild-type control mice, suggesting that $100 \mu \mathrm{g} / \mathrm{kg}$ TPL may be toxic to the mice, and thus have caused them to lose weight.

Levels of total cholesterol and total triglyceride are highly increased in ApoE\% mice models. Atherosclerosis is inextricably linked with abnormal lipid metabolism. Abnormal lipid metabolism is mainly characterized by higher levels of plasma cholesterol, which is an important risk factor for the injury induced by atherosclerosis $(27,28)$. In the present study, the levels of total cholesterol and total triglyceride in $\mathrm{ApoE}^{-/}$mice and normal control mice were measured. As shown in Fig. 3, after 1 month of high-fat diet, the total cholesterol and total triglyceride levels were gradually and significantly increased in the $\mathrm{ApoE}^{-/-}$mice compared with those in wild-type mice

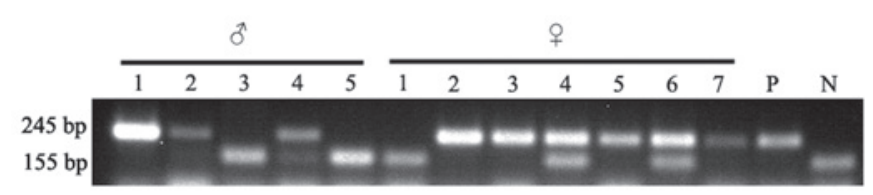

Figure 1. ApoE ${ }^{\%}$ mice were identified and selected by polymerase chain reaction (PCR). The tails from 5 male $(ð)$ C57BL/6 mice and 7 female $(+\circ)$ mice were cut off, and the DNA samples were extracted. Three primers were used to detect the genotype of the ApoE gene. Genotypes of ApoE in the 12 mice were detected by PCR assay. A positive (P) sample for ApoE $\%$ mice and a negative (N) DNA sample (wild type) were provided as controls. ApoE, apolipoprotein $\mathrm{E}$.

$(\mathrm{P}<0.05$ and $\mathrm{P}<0.01$, respectively), and the difference between the levels in the two groups increased as the duration of the high-fat diet extended $(\mathrm{P}<0.01)$. These data indicate that the atherosclerotic mouse model was successfully constructed.

Treatment with TPL contributes to the downregulation of total cholesterol and total triglyceride levels in ApoE $\%$ mice. In order to detect the effects of TPL on the production of total cholesterol and total triglyceride, various concentrations of TPL were used to treat $\mathrm{ApoE}^{-/}$mice fed with a high-fat diet. From week 8 after birth, mice with an $\mathrm{ApoE}^{-/}$genotype were fed a high-fat diet, and treated with 25,50 or $100 \mu \mathrm{g} / \mathrm{kg}$ TPL. The total cholesterol and total triglyceride levels were detected at 16 and 20 weeks, respectively. The results demonstrated that the levels of total cholesterol and total glyceride were decreased by TPL treatment in a dose-dependent manner (Fig. 4).

TPL inhibits the secretion of inflammatory cytokines in macrophages. Foam cells, which are fat-laden macrophages, are the characteristic pathological cells in atherosclerotic plaques, and are mainly derived from blood mononuclear cells and vascular smooth muscle cells (29). Whether TPL is able to inhibit cytokine production in macrophages during the progression of atherosclerosis was investigated in the present study. As shown in Fig. 5, macrophages from ApoE ${ }^{-/}$mice produced high levels of the inflammatory cytokines TNF- $\alpha$, IL-1 $\beta$, IL-6 and IL-8. However, treatment with TPL at concentrations of 25, 50 and $100 \mu \mathrm{g} / \mathrm{kg}$ markedly reduced the levels of the cytokines in a dose-dependent manner, which demonstrated that TPL inhibited the progression of inflammation.

TPL treatment increases the expression of ABCAl but has no effect on liver $X$ receptor $\alpha(L X R \alpha)$. ABCA1 is a membrane-associated protein and mediates the transmission of the intracellular cholesterol. It contributes to reducing the formation of foam cells and preventing the occurrence of atherosclerosis (30). The expression of ABCA1 can be induced by increased levels of HDL stimulated by LXR and retinoid X receptor (30). The expression levels of LXR $\alpha$ and ABCA1 were determined in the present study. As shown in Fig. 6, the expression levels of ABCA1 in the macrophages of $\mathrm{ApoE}^{-/}$mice were significantly increased in the mice treated with TPL. However, the expression levels of LXR $\alpha$ were not significantly affected by treatment with different concentrations of TPL. 


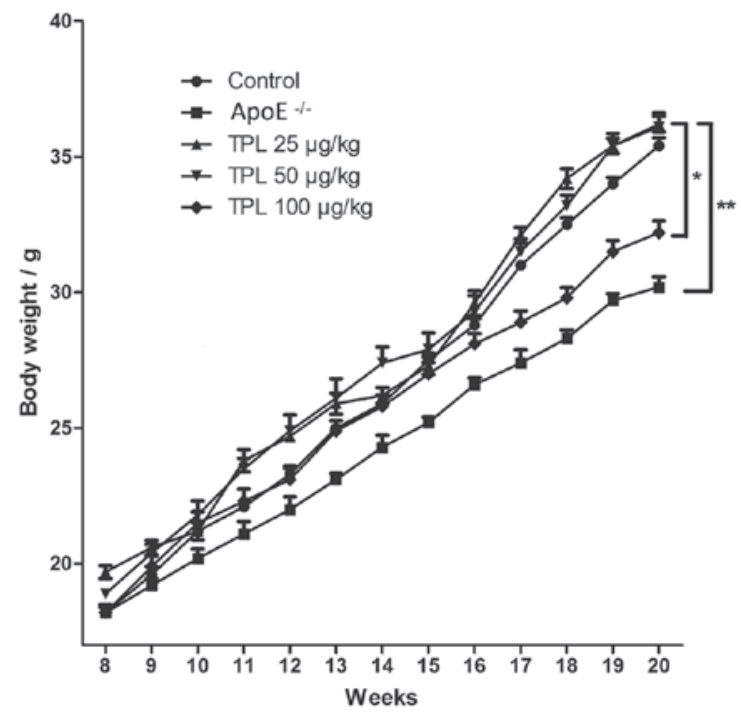

Figure 2. Body weights of ApoE $/$ knock-out mice were less than those of the other groups. ApoE $/$ knock-out mice were given a high-fat diet and treated with 25,50 or $100 \mu \mathrm{g} / \mathrm{kg}$ TPL every twice day. Body weights were measured once every week prior to injection. ApoE $/ 1$ mice without any treatment were used as a positive control (ApoE $\%$ group) and wild-type $\mathrm{C} 57 \mathrm{BL} / 6$ mice with the same genetic background were the negative control (control group). ${ }^{*} \mathrm{P}<0.05$ and ${ }^{* *} \mathrm{P}<0.01$ vs. the control group. ApoE, apolipoprotein E. TPL, triptolide.

A

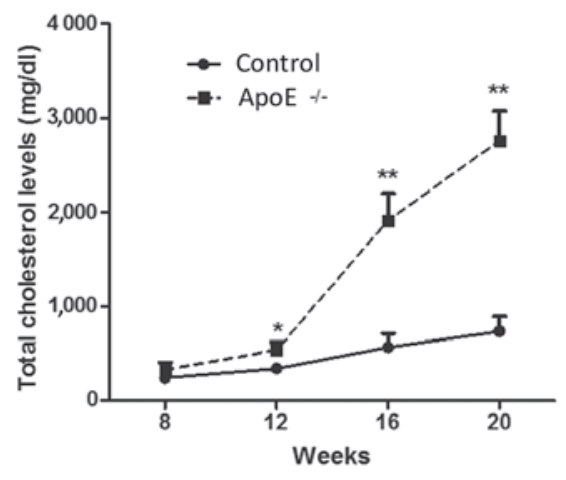

B

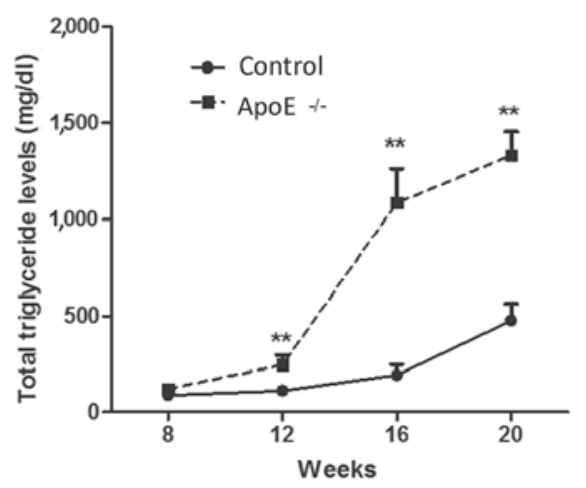

Figure 3. Levels of total cholesterol and total triglyceride are highly increased in ApoE $\%$ mice models. ApoE $\%$ mice and wild-type (control) mice were given a high-fat diet from week 8 after birth. At 16 and 20 weeks, fasted blood (A) total cholesterol and (B) total triglyceride levels were measured after $12-16 \mathrm{~h}$ of fasting. ${ }^{*} \mathrm{P}<0.05$ and ${ }^{* *} \mathrm{P}<0.01$ vs. the control group. ApoE, apolipoprotein E.

\section{Discussion}

Atherosclerosis is the main pathology for cardiovascular
A

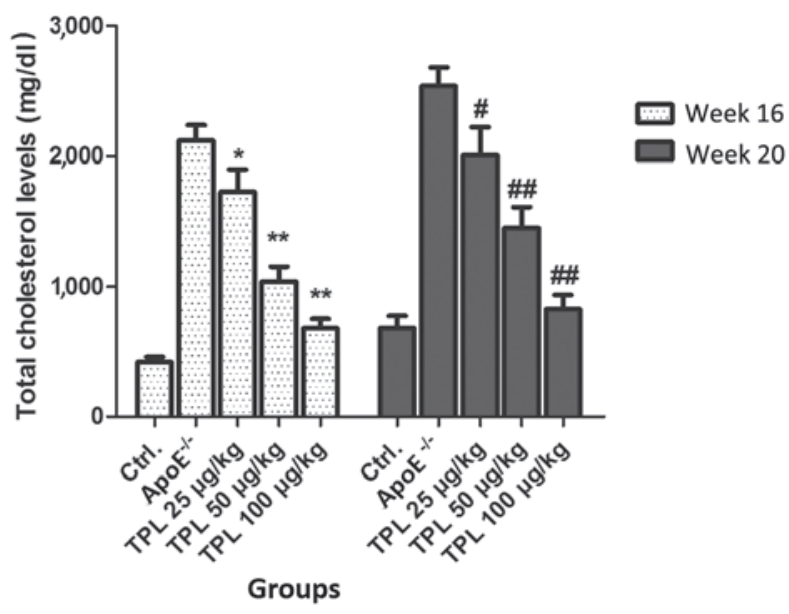

B

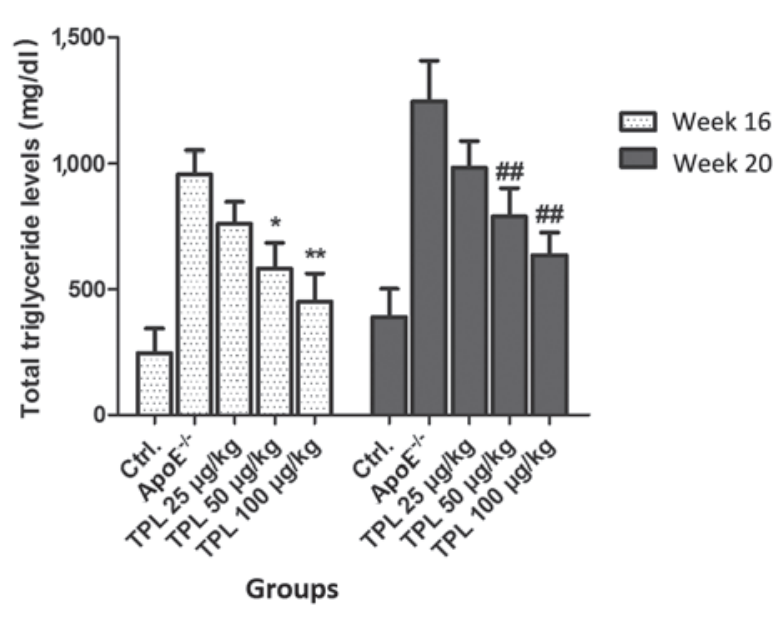

Figure 4. Treatment with TPL contributes to the downregulation of total cholesterol and total triglyceride levels in $\mathrm{ApoE} \%$ mice. $\mathrm{ApoE} \%$ mice were given a high-fat diet from week 8 after birth and were treated with 25,50 or $100 \mu \mathrm{g} / \mathrm{kg}$ TPL every 2 days. At weeks 16 and 20, fasted blood levels of (A) total cholesterol and (B) total triglyceride were measured after $12-16 \mathrm{~h}$ of fasting. ${ }^{*} \mathrm{P}<0.05$ and ${ }^{* * *} \mathrm{P}<0.01$ vs. untreated ApoE $/$ mice at 16 weeks. ${ }^{~} \mathrm{P}<0.05$ and ${ }^{\# \#} \mathrm{P}<0.01$ vs. untreated $\mathrm{ApoE} / /$ mice at 20 weeks. ApoE, apolipoprotein E; TPL, triptolide; Ctrl., control (wild-type mice).

and cerebrovascular diseases, and it progresses with chronic inflammation (31-33). TPL is a compound originally extracted from the plant $T$. wilfordii Hook.f., which has potent anti-inflammatory activity (34). The aim of the present study was to identify whether TPL was able to inhibit the progression of atherosclerosis by an anti-inflammatory mechanism. Thus, ApoE $\%$ mice were given high-fat diet to construct animal models of atherosclerosis, and different doses of TPL were used to treat the mice. The observation and determination of the effects of TPL in the progression of atherosclerosis may provide new insights useful for the therapy of atherosclerotic disease.

Lipoproteins are predominantly synthesized in the liver and intestines. ApoE is a type of apolipoprotein found in VLDL, CMs and their debris; it is a ligand of the LDL receptor in the body and has an important role in lipid metabolism. ApoE deficiency can cause the accumulation of cholesterol-rich lipoproteins in the blood, which induces the formation and development of atherosclerotic lesions (35). Abnormal lipid metabolism, in particular that leading to high levels of plasma 
A

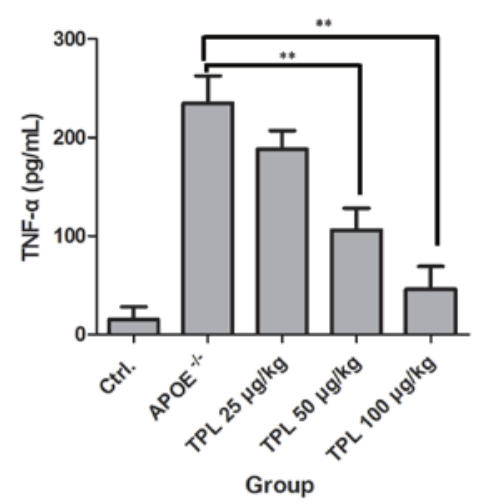

C

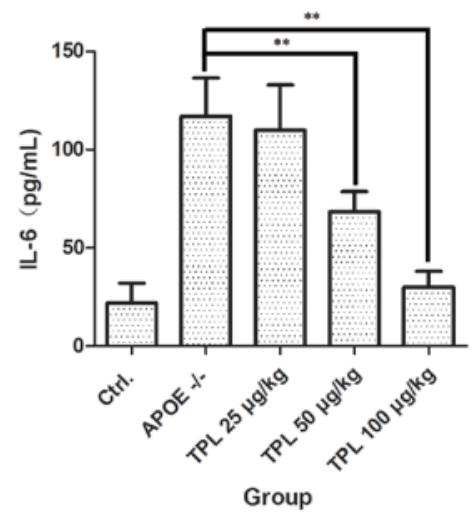

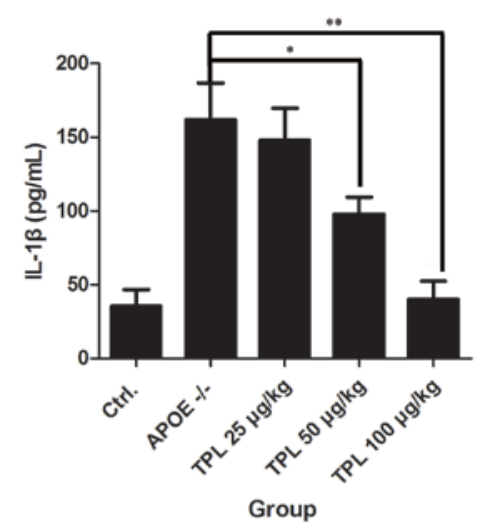

D

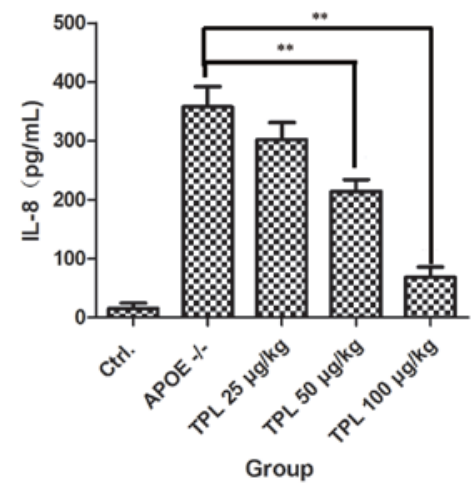

Figure 5. TPL inhibits the secretion of inflammatory cytokines in macrophages. ApoE $\%$ mice were given a high-fat diet from week 8 after birth and then treated with 25,50 or $100 \mu \mathrm{g} / \mathrm{kg}$ TPL every 2 days. At week 16, macrophages from the different groups were prepared and stimulated with $25 \mu \mathrm{g} / \mathrm{ml}$ oxidized low-density lipoprotein. The secretion levels of the inflammatory cytokines (A) TNF- $\alpha$, (B) IL-1 $\beta$, (C) IL-6 and (D) IL-8 were determined using enzyme-linked immunosorbent assay kits. ${ }^{*} \mathrm{P}<0.05$ and ${ }^{* *} \mathrm{P}<0.01$ vs. untreated ApoE $/$ mice. ApoE, apolipoprotein E; TPL, triptolide; Ctrl., control (wild-type mice); TNF, tumor necrosis factor; IL, interleukin.

A

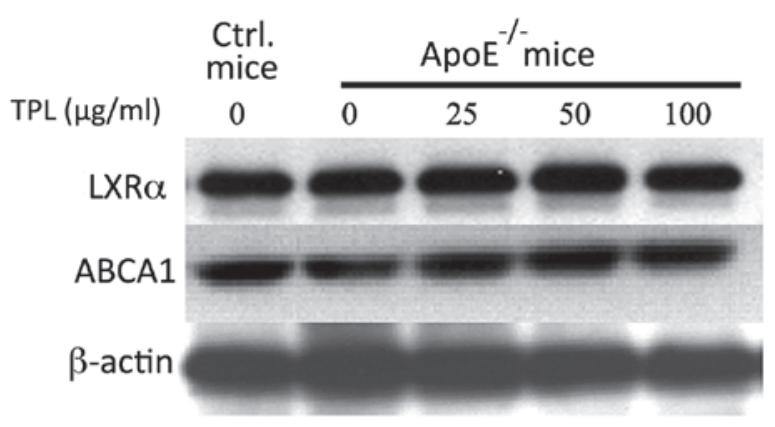

B

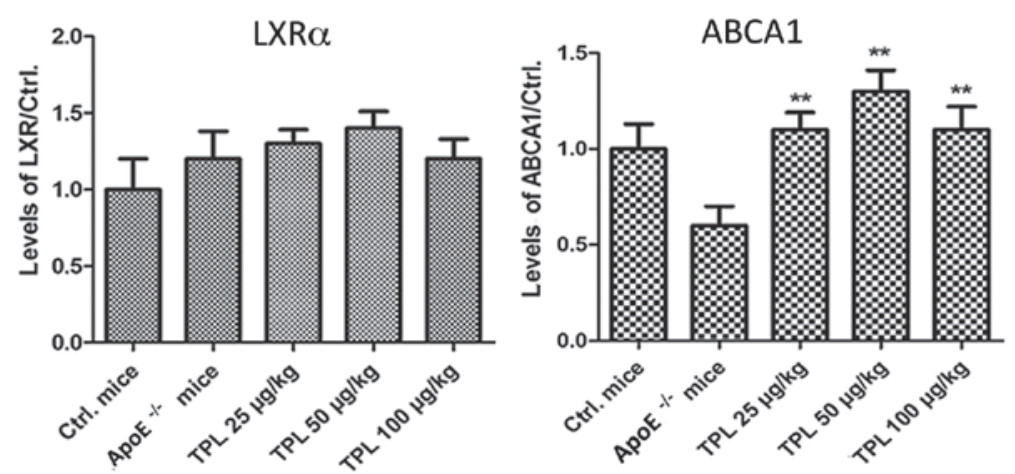

Figure 6. Triptolide treatment increases the expression of ABCA1 but has no effect on LXR $\alpha$. ApoE $\%$ mice were given a high-fat diet from week 8 after birth and then treated with 25,50 or $100 \mu \mathrm{g} / \mathrm{kg}$ TPL every 2 days. At week 16, macrophages from the different group were prepared. (A) Expression levels of LXR $\alpha$ and $\mathrm{ABCA} 1$ were determined by western blotting. (B) Expression levels of LXR $\alpha$ and ABCA1 determined by capturing the gray value of every band and analyzing them using imageJ software; the expression ratios LXR $\alpha / C$ trl. and ABCA1/Ctrl. are shown. Expression levels of LXR $\alpha$ and ABCA1 in wild-type mice were used as controls. ${ }^{* *} \mathrm{P}<0.01$ vs. the ApoE $\%$ group. ApoE, apolipoprotein E; TPL, triptolide; Ctrl., control (wild-type mice); ABCA1, anti-ATP-binding cassette transporter A1; LXR $\alpha$, liver X receptor $\alpha$. 
cholesterol, is an important risk factor for the development of atherosclerosis (36). In the present study, ApoE gene knock-out mice were used the animal models to explore the mechanism of atherosclerosis.

TPL has been demonstrated to be the main active component of the herb T. wilfordii Hook.f., which is used in traditional Chinese medicine, and it has been revealed to exert anti-inflammatory activity by inhibiting the NF- $\kappa \mathrm{B}$ signaling pathway (25). Lee et al found that TPL confers neuroprotection against traumatic brain injury, at least in part, via its anti-inflammatory activity (37). Han et al demonstrated that TPL contributes to the treatment of psoriasis and other immune-mediated inflammatory diseases (38). An autoimmune disease, rheumatoid arthritis, is associated with increased production of pro-inflammatory cytokines. Matta et al found that TPL alleviated the progression of rheumatoid arthritis not only by inhibiting NF- $\mathrm{KB}$-regulated reporter transcription, but also by blocking the activity of other transcription factors (39). Atherosclerosis is also a disease associated with chronic inflammation. In the present study, various concentrations of TPL were used to treat ApoE $/$ mice fed with a high-fat diet for longer than 2 months. The results demonstrated that TPL inhibited the secretion of inflammatory cytokines in $\mathrm{ApoE}^{-/}$mice, which was consistent with the previous studies and confirmed that TPL has anti-inflammatory activity.

In conclusion, the results of the present study demonstrate that the abnormal lipid metabolism and a chronic inflammatory response contributed to the progression of atherosclerosis in ApoE $\%$ mice, and that treatment with TPL regulated lipid metabolism and inhibited inflammation in $\mathrm{ApoE}^{-/}$mice. The clarification of the mechanism of atherosclerosis and the effects of TPL is helpful and instructive in the clinical treatment of this disease.

\section{References}

1. Watanabe T, Sato K, Itoh F, Iso Y, Nagashima M, Hirano T and Shichiri M: The roles of salusins in atherosclerosis and related cardiovascular diseases. J Am Soc Hypertens 5: 359-365, 2011.

2. Zeng Y, Song JX and Shen XC: Herbal remedies supply a novel prospect for the treatment of atherosclerosis: A review of current mechanism studies. Phytother Res 26: 159-167, 2012.

3. Suzuki M, Minami A, Nakanishi A, Kobayashi K, Matsuda S, Ogura Y and Kitagishi Y: Atherosclerosis and tumor suppressor molecules (review). Int J Mol Med 34: 934-940, 2014.

4. Hulthe J, Wikstrand J, Mattsson-Hulten L and Fagerberg B Circulating ICAM-1 (intercellular cell-adhesion molecule 1) is associated with early stages of atherosclerosis development and with inflammatory cytokines in healthy 58 year-old men: The atherosclerosis and insulin resistance (AIR) study. Clin Sci (Lond) 103: 123-129, 2002

5. Maranhão RC and Leite AC Jr: Development of anti-atherosclerosis therapy based on the inflammatory and proliferative aspects of the disease. Curr Pharm Des 21: 1196-1204, 2015.

6. Shing CM, Fassett RG, Peake JM and Coombes JS: Voluntary exercise decreases atherosclerosis in nephrectomised ApoE knockout mice. PloS One 10: e0120287, 2015.

7. González-Gay MA, González-Juanatey C, Llorca J and Castañeda S: The influence of inflammation in the development of subclinical atherosclerosis in psoriatic arthritis: Comment on 'Cardiovascular comorbidities in patients with psoriatic arthritis: A systematic review' by Jamnistki et al: Ann Rheum Dis 73: e27, 2014.

8. Hansson GK: Immune and inflammatory mechanisms in the development of atherosclerosis. Br Heart J 69 (Suppl): S38-S41, 1993.
9. Pizzi J, Silva LR, Moser D and Leite N: Relationship between subclinical atherosclerosis, blood pressure and lipid profile in obese children and adolescents: A systematic review. Arq Bras Endocrinol Metabol 57: 1-6, 2013.

10. Kreisberg RA and Oberman A: Clinical review 141: Lipids and atherosclerosis: Lessons learned from randomized controlled trials of lipid lowering and other relevant studies. J Clin Endocrinol Metab 87: 423-437, 2002.

11. Zhang L and Yang L: Anti-inflammatory effects of vinpocetine in atherosclerosis and ischemic stroke: A review of the literature. Molecules 20: 335-347, 2014.

12. Ross R: Atherosclerosis is an inflammatory disease. Am Heart J 138: S419-S420, 1999.

13. Ross R: Atherosclerosis - an inflammatory disease. N Engl J Med 340: 115-126, 1999.

14. Schlag B, Winkler L, Plonné D, Dürer U and Dargel R: Preparation of apoE-free rat low density lipoprotein for catabolic studies. J Lipid Res 28: 1521-1524, 1987.

15. Martinez-Oliván J, Arias-Moreno X, Velazquez-Campoy A, Millet $\mathrm{O}$ and Sancho J: LDL receptor/lipoprotein recognition: Endosomal weakening of ApoB and ApoE binding to the convex face of the LR5 repeat. FEBS J 281: 1534-1546, 2014.

16. Kostogrys RB, Franczyk-żarów M, Maślak E, Gajda M, Mateuszuk L, Jackson CL and Chłopicki S: Low carbohydrate, high protein diet promotes atherosclerosis in apolipoprotein E/low-density lipoprotein receptor double knockout mice (apoE/LDLR $/$ ). Atherosclerosis 223: 327-331, 2012.

17. Shimano H, Yamada N, Katsuki M, Yamamoto K, Gotoda T, Harada K, Shimada M and Yazaki Y: Plasma lipoprotein metabolism in transgenic mice overexpressing apolipoprotein E. Accelerated clearance of lipoproteins containing apolipoprotein B. J Clin Invest 90: 2084-2091, 1992.

18. Shimano H, Yamada N, Katsuki M, Shimada M, Gotoda T, Harada K, Murase T, Fukazawa C, Takaku F and Yazaki Y: Overexpression of apolipoprotein $\mathrm{E}$ in transgenic mice: Marked reduction in plasma lipoproteins except high density lipoprotein and resistance against diet-induced hypercholesterolemia. Proc Natl Acad Sci USA 89: 1750-1754, 1992.

19. Dallongeville J, Lussier-Cacan S and Davignon J: Modulation of plasma triglyceride levels by apoE phenotype: A meta-analysis. J Lipid Res 33: 447-454, 1992.

20. Zhang SH, Reddick RL, Piedrahita JA and Maeda N: Spontaneous hypercholesterolemia and arterial lesions in mice lacking apolipoprotein E. Science 258: 468-471, 1992.

21. Weingärtner O, Ulrich C, Lütjohann D, Ismail K, Schirmer SH, Vanmierlo T, Böhm M and Laufs U: Differential effects on inhibition of cholesterol absorption by plant stanol and plant sterol esters in apoE $\mathrm{E}^{-}$mice. Cardiovasc Res 90: 484-492, 2011.

22. Sato M, Yamada Y, Matsuoka H, Nakashima S, Kamiya T, Ikeguchi $\mathrm{M}$ and Imaizumi K: Dietary pine bark extract reduces atherosclerotic lesion development in male ApoE-deficient mice by lowering the serum cholesterol level. Biosci Biotechnol Biochem 73: 1314-1317, 2009

23. Gopal K, Kumar K, Nandini R, Jahan P and Kumar MJ: High fat diet containing cholesterol induce aortic aneurysm through recruitment and proliferation of circulating agranulocytes in apoE knock out mice model. J Thromb Thrombolysis 30: 154-163, 2010.

24. Zou Y, Du H, Yin M, Zhang L, Mao L, Xiao N, Ren G, Zhang C and Pan J: Effects of high dietary fat and cholesterol on expression of PPAR alpha, LXR alpha and their responsive genes in the liver of apoE and LDLR double deficient mice. Mol Cell Biochem 323: 195-205, 2009.

25. Wang X, Zhang L, Duan W, Liu B, Gong P, Ding Y and Wu X: Anti-inflammatory effects of triptolide by inhibiting the $N F-\kappa B$ signalling pathway in LPS-induced acute lung injury in a murine model. Mol Med Rep 10: 447-452, 2014.

26. Chen BJ: Triptolide, a novel immunosuppressive and anti-inflammatory agent purified from a Chinese herb Tripterygium wilfordii Hook F. Leuk Lymphoma 42: 253-265, 2001.

27. Calabresi L, Gomaraschi M, Simonelli S, Bernini F and Franceschini G: HDL and atherosclerosis: Insights from inherited HDL disorders. Biochim Biophys Acta 1851: 13-18, 2015.

28. Rao X, Zhong J, Maiseyeu A, Gopalakrishnan B, Villamena FA, Chen LC, Harkema JR, Sun Q and Rajagopalan S: CD36-dependent 7-ketocholesterol accumulation in macrophages mediates progression of atherosclerosis in response to chronic air pollution exposure. Circulation Res 115: 770-780, 2014. 
29. Woollard KJ: Immunological aspects of atherosclerosis. Clinical Sci 125: 221-235, 2013.

30. Chen JH, Wang CJ, Wang CP, Sheu JY, Lin CL and Lin HH: Hibiscus sabdariffa leaf polyphenolic extract inhibits LDL oxidation and foam cell formation involving up-regulation of LXRalpha/ABCA1 pathway. Food Chem 141: 397-406, 2013.

31. Wang W, Lee Y and Lee CH: Review: The physiological and computational approaches for atherosclerosis treatment. Int $\mathrm{J}$ Cardiol 167: 1664-1676, 2013.

32. Rodondi N, Auer R, de Bosset Sulzer V, Ghali WA and Cornuz J: Atherosclerosis screening by noninvasive imaging for cardiovascular prevention: A systematic review. J Gen Intern Med 27: 220-231, 2012.

33. Hartman J and Frishman WH: Inflammation and atherosclerosis: A review of the role of interleukin- 6 in the development of atherosclerosis and the potential for targeted drug therapy. Cardiol Rev 22: 147-151, 2014.
34. Ziaei S and Halaby R: Immunosuppressive, anti-inflammatory and anti-cancer properties of triptolide: A mini review. Avicenna J Phytomed 6: 149-164, 2016.

35. Hoeke G, Kooijman S, Boon MR, Rensen PC and Berbee JF: Role of brown fat in lipoprotein metabolism and atherosclerosis. Circ Res 118: 173-182, 2016.

36. Smith BW, Miller RJ, Wilund KR, O'Brien WD Jr and Erdman JW Jr Effects of tomato and soy germ on lipid bioaccumulation and atherosclerosis in ApoE-/- mice. J Food Sci 80: H1918-1925, 2015.

37. Lee HF, Lee TS and Kou YR: Anti-inflammatory and neuroprotective effects of triptolide on traumatic brain injury in rats. Respir Physiol Neurobiol 182: 1-8, 2012.

38. Han R, Rostami-Yazdi M, Gerdes S and Mrowietz U: Triptolide in the treatment of psoriasis and other immune-mediated inflammatory diseases. Br J Clin Pharmacol 74: 424-436, 2012.

39. Matta R, Wang X, Ge H, Ray W, Nelin LD and Liu Y: Triptolide induces anti-inflammatory cellular responses. Am J Transl Res 1: 267-282, 2009. 